\title{
EFEITOS DA PREMATURIDADE SOBRE O DESENVOLVIMENTO DE LACTENTES
}

\section{EFFECTS OF THE PREMATURITY ON THE DEVELOPMENT OF LACTENTES}

Olga Maria Piazentin Rolim Rodrigues ${ }^{1}$

Alessandra Turini Bolsoni-Silva ${ }^{1}$

Rodrigues OMPR; Bolsoni-Silva AT. Efeitos da prematuridade sobre o desenvolvimento de lactentes. Rev. Bras. Cresc. e Desenv. Hum. 2011; 21(1): 111-121.

\section{Resumo:}

Foi avaliado o desenvolvimento de lactentes, correlacionando-o com a prematuridade. Participaram do estudo 130 lactentes que foram avaliados mensalmente no decorrer do primeiro ano de vida com o Inventário Portage Operacionalizado. Desses, 56 eram sem condição de risco identificados por ocasião do nascimento (Grupo 1) e 74 eram nascidos prematuros (Grupo 2), até 37 semanas de gestação. Os resultados obtidos apontaram que os lactentes do Grupo 2 apresentaram desempenhos significantemente inferior aos do Grupo 1 em $68 \%$ das análises conduzidas, com destaque para as áreas de desenvolvimento motor, cognição e socialização. Tais resultados são condizentes com os obtidos na literatura que apontam a prematuridade como uma das condições proximais mais prejudiciais ao desenvolvimento de lactentes.

Palavras-chave: inventário Portage operacionalizado; desenvolvimento de lactentes; avaliação infantil; prematuridade.

\section{Abstract:}

The aim of this study was to evaluate the behavioral aspects of infants relating them to prematurity. A total of 130 lactentes were evaluated every month during the first year of life, employing the Operational Portage Inventory . Group 1, 56 newborns with no life risk at birth and Group 2,74 premature babies, those that was born until 37 gestation weeks. The outcomes obtained showed that the premature babies (Group 2) presented a totally different performance than Group 1 in $68 \%$ of the analyses made, especially for motor development, cognition and socialization. The outcomes are in accord to the ones obtained in the literature, which indicate prematurity as one of the most harmful proximal conditions for the development of infants.

Key words: operational portage inventory; development of lactentes; infantile evaluation; prematurity.

1 Departamento de Psicologia, Faculdade de Ciências, Universidade Estadual Paulista Julio de Mesquita Filho (UNESP), Bauru. Correspondência para: Olga Maria Piazentin Rolim Rodrigues. Departamento de Psicologia, Faculdade de Ciências, Universidade Estadual Paulista Julio de Mesquita Filho (UNESP), Bauru. Av. Luiz Edmundo C. Coube, 14-01 Bairro Vargem Limpa. Bauru/SP. CEP: 17018-110. Fone: (14) 3103 6087/3103 6090.

Parte da Tese de Livre Docência da primeira autora, defendida em setembro de 2009, na Faculdade de Ciências, Universidade Estadual Paulista Julio de Mesquita Filho (UNESP), Bauru, com o título: O Inventário Portage Operacionalizado e o Desenvolvimento De Bebês. 


\section{INTRODUÇÃO}

O desenvolvimento humano é um processo contínuo de aprendizagem, resultado de interações recíprocas entre o ambiente e o indivíduo que se modificam, implicando reorganizações constantes deste sistema indivíduo-ambiente'. Nessa perspectiva, as interações indivíduo-ambiente incluem fatores biológicos, genéticos, sociais e culturais que agem simultaneamente sobre o desenvolvimento. Todavia, ainda que se identifique a presença de algum fator de risco, a continuidade dos seus efeitos no processo de desenvolvimento não é linear, considerando que este é resultado da interação do indivíduo com o ambiente e comportamentos podem emergir, modificar e, até mesmo, desaparecer ${ }^{2}$.

Alguns comportamentos, se presentes no repertório do lactente, facilitam seu acesso a outras possibilidades interativas disponíveis no seu ambiente, maximizando a aprendizagem de novos comportamentos relevantes para o indivíduo e/ou para o ambiente em que está inseri$\mathrm{do}^{3}$, mediados pela mãe ou outro adulto significativo. $\mathrm{O}$ ambiente, nas múltiplas influências exercidas nas interações estabelecidas pelo indivíduo, tem, portanto, papel importante no desenvolvimento de repertórios comportamentais. Esses fatores ambientais podem ter função de risco ou de proteção ao desenvolvimento da criança.

Matos $^{4}$ e Oliveira ${ }^{5}$ consideram que os fatores de risco podem ser distais, meioproximais e proximais. Entre os fatores distais estão os indicadores de risco social, como a baixa renda. Os meio-proximais seriam os mecanismos de risco familiar e, entre eles, os estilos e práticas parentais, eventos familiares estressantes e rede de apoio pobre. Os proximais envolveriam os mecanismos de risco individualizado, como condições de saúde do recém-nascido(RN). Por outro lado, fatores de proteção são aqueles que podem minimizar o impacto dos fatores de risco ${ }^{5}$, por exemplo uma mãe que possui renda familiar baixa (fator de risco) pode promover o desenvolvimento de seu filho oferecendo ambiente estimulador e práticas educativas adequadas.

Atrasos no desenvolvimento são produzidos, então, pela combinação de fatores de risco genéticos, biológicos, psicológicos e ambientais, envolvendo interações complexas entre eles. Há consenso entre pesquisadores de que o efeito cumulativo de risco é mais importante na determinação de problemas emocionais da criança do que a presença de um estressor único, independentemente de sua magnitude ${ }^{6,7,8}$. Os efeitos de tais fatores probabilísticos são avaliados comparando, posteriormente, o desenvolvimento da criança com o da população em geral, no caso da utilização de instrumentos padronizados, ou com o desempenho de grupos controle em que os fatores de risco não foram identificados.

A Análise do Comportamento, ainda que se paute por análises idiossincráticas e menos pelas nomotéticas, não desconsidera a influência probabilística dessas variáveis ${ }^{9}$. Para estes autores, ainda que limitados, os modelos de avaliações nomotéticas (baseadas em medidas múltiplas de grandes amostras) proveem ao analista do comportamento um conjunto de variáveis causais possíveis, que podem ser ou não funcionais para determinados lactentes. Pesquisadores atestam que este tipo de avaliação pode subsidiar intervenções ${ }^{10,9}$ e mapear variáveis disposições do ambiente e do indivíduo que podem tornar mais ou menos provável a ocorrência da interação com o ambiente ${ }^{11}$.

Resegue, Puccini e Silva ${ }^{8}$ destacam que os avanços científicos apontam para a plasticidade do cérebro humano, que é mais acentuada nos primeiros anos de vida e suscetível à estimulação. Tais avanços reforçam a possibilidade de evolução de crianças com prognóstico de alterações no desenvolvimento que tem sido otimizada pelo diagnóstico e pela intervenção precoce. Entre os fatores de risco para o desenvolvimento infantil, Resegue et al. ${ }^{7,8}$ destacam a prematuridade. 
Prematuros ou pré-termos são considerados os recém-nascidos com idade gestacional inferior a 37 semanas, o que resulta num grupo heterogêneo de crianças com idades próximas ao termo, ou ainda aquelas com idades gestacionais abaixo de 32 semanas. O CID- $10^{12}$ define idade gestacional: termo - de 37 a 42 semanas de gestação (de 260 a 293 dias); prétermo - menos de 37 semanas (menos de 259 dias); pós-termo - 42 semanas ou mais (mais de 293 dias). Quanto menor a idade gestacional, maior a probabilidade de risco para a mortalidade, abandono, problemas de saúde etc.

Rugolo ${ }^{13}$ mostra que, no Brasil, a exemplo dos países desenvolvidos, tem aumentado a taxa de sobrevivência de recém-nascidos considerados de extrema prematuridade, aqueles na faixa de 500 a 1000 gramas, mas também alerta para " ... o elevado custo da assistência neonatal e os custos econômicos e sociais dos cuidados pós-alta aos recém-nascidos sequelados" (p. 114).

As avaliações de desenvolvimento nos anos iniciais, para avaliar os efeitos da prematuridade, podem ser conduzidas considerando a idade cronológica dos RNs ou a idade corrigida. A idade corrigida é a idade pós-natal, menos o número de semanas que faltou entre o nascimento prematuro e o referencial de 40 semanas $^{14}$.

Rugolo $^{13}$ defende o uso da idade corrigida em casos de prematuridade extrema, como uma forma de não prejudicar a avaliação do desenvolvimento do lactente. Todavia, parece que não há consenso entre os pesquisadores. Manacero e Nunes ${ }^{15}$ avaliaram o desempenho motor de 44 RNs prematuros (entre 32 e 34 semanas), aos quatro e oito meses de idade corrigida, pela Escala Motora Infantil Alberta (AIMS) e compararam com o peso ao nascer. Os resultados mostraram desenvolvimento motor na sequência esperada e não observaram relação com o peso ao nascer. Batista ${ }^{16}$, em um estudo conduzido com 21 prematuros, durante o primeiro ano de vida, constatou que, por volta de três meses, $77 \%$ deles apresentaram uma recuperação importante no seu desenvolvimento com repertório comportamental próximos à sua idade cronológica.

Pesquisadores têm apontado que uma das consequências da prematuridade é o atraso do desenvolvimento motor, cujos efeitos aparecem nas idades posteriores, em dificuldades na realização de atividades de vida diária e nas atividades acadêmicas sendo que, nas últimas, podem comprometer o desempenho escolar $^{17,18,19,20}$. É possível que na faixa etária até um ano de idade, os comportamentos motores sejam pontos comportamentais que propiciam o desenvolvimento de outros comportamentos.

Outras consequências da prematuridade em idades posteriores têm sido estudadas em populações de crianças nascidas prematuras, como o desenvolvimento geral ${ }^{21,22,23}$, ou em áreas específicas, como o desenvolvimento social $^{24,25}$, o desempenho intelectual ${ }^{24,25,26} \mathrm{e} \mathrm{o}$ de linguagem ${ }^{25,27}$.

Os estudos analisados permitem concluir que a prematuridade pode trazer prejuízos para o desenvolvimento infantil. Todavia, não há consenso quanto ao uso da idade corrigida para a avaliação dos efeitos da prematuridade.

Os instrumentos como testes, escalas ou inventários, usados para avaliar o desenvolvimento de lactentes, têm sido utilizados em pesquisas aplicadas, clínicas e estabelecimentos educacionais, com relativo poder preditivo, ou para subsidiar a implementação de programas de estimulação precoce, orientando o planejamento de ações pontuais com crianças e seus cuidadores $^{28}$.

O Inventário Portage Operacionalizado ${ }^{29}$ tem se mostrado útil para avaliar o desenvolvimento infantil e sugere a observação de comportamentos de crianças sob determinadas condições cuidadosamente organizadas ou a partir do relato dos cuidadores ${ }^{30,31}$. Identificar áreas em defasagem no repertório comportamental 
de RNs prematuros nas áreas propostas pelo inventário (cognição, desenvolvimento motor, autocuidado, socialização e linguagem) pode subsidiar procedimentos de intervenção precoce específico para essa população.

Desta maneira, o estudo tem por objetivo avaliar o repertório comportamental de lactentes e correlacionar com o evento da prematuridade.

\section{MÉTODO}

\section{Participantes}

Participaram do estudo 130 RNs, que foram avaliados no decorrer do primeiro ano de vida. Desses, 56 eram sem condições de risco identificadas por ocasião do nascimento (Grupo 1) e 74 eram nascidos prematuros (Grupo 2), até 37 semanas de gestação, de acordo com a definição do CID- $10^{12}$. No Grupo 1, 48\% eram meninos e no Grupo 2, 53\%.

\section{Material}

Foi utilizado um roteiro de entrevista (Entrevista Inicial) para a coleta de dados demográficos, incluindo o tempo de gestação.

O desenvolvimento dos lactentes foi avaliado a partir do Inventário Portage Operacionalizado (IPO ${ }^{29}$ contendo, para a faixa etária de zero a 12 meses, 154 itens que compõem a escala de desenvolvimento geral, sendo 13 para avaliação de linguagem, 18 para autocuidado, 30 para cognição, 34 para socialização e 59 para desenvolvimento motor. O IPO é um instrumento não padronizado tendo sido operacionalizado. Ele prevê que os comportamentos dos lactentes sejam, na medida do possível, observados a partir da organização de contingências que possibilitem, na situação de teste, fazê-los. Há, no IPO, um rol de comportamentos que devem ser especificamente observados. Todavia, para alguns deles o relato do observador deve ser considerado.
Brinquedos e instrumentos de acordo com as especificações do manual compunham o material utilizado nas avaliações.

\section{Procedimentos de coleta de dados}

Os pais das crianças nascidas a partir de abril de 2006, em uma maternidade pública eram convidados a participar do projeto, "Acompanhamento do desenvolvimento de crianças: avaliação e orientação aos pais". Nessa ocasião, ficava agendado o primeiro encontro, em dia próximo à data de aniversário de um mês, para a primeira avaliação, no Centro de Psicologia Aplicada de uma universidade pública do interior paulista.

$\mathrm{O}$ primeiro encontro era iniciado pela apresentação do projeto, especificando seus objetivos e a necessidade de ocorrência de avaliações subsequentes, mês a mês, até o primeiro aniversário da criança. Em seguida aplicava-se a Entrevista Inicial e era conduzida a primeira avaliação do repertório comportamental dos lactentes, de acordo com as instruções do manual de aplicação do IPO $^{29}$, adequadas ao novo protocolo. Ainda nesse encontro, era agendada a próxima avaliação por ocasião do aniversário de segundo mês do bebê e, assim, era feito o mesmo procedimento para as avaliações subsequentes.

As avaliações foram conduzidas na presença de um ou ambos os genitores que serviram como mediadores e informantes. $\mathrm{O}$ adulto (pai ou mãe), nesta fase, pode servir como modelo e mediador, garantindo, às vezes, que a atividade se realize. Neste caso, as orientações são dadas de forma a garantir que a situação teste possibilitasse a resposta do lactente.

O comportamento da criança foi registrado a partir de estimulação ambiental (por exemplo: Alcança um objeto colocado na sua frente (distância de 15 a $20 \mathrm{~cm}$ ), da emissão de comportamentos espontâneos (por exemplo: Olha para a própria mão, frequentemente, sorrindo, vocalizando) e do relato dos pais em função de questionamentos feitos pelas 
aplicadoras, em se tratando de comportamentos não observáveis na situação de teste (comportamentos relacionados à alimentação, sobre a rotina do sono, choro, se reconhece os membros da família etc.).

Procedimentos de tratamento e análise de dados

As idades consideradas para os dois grupos se referem à idade cronológica dos recém-nascidos por ocasião da avaliação.

Os dados foram analisados estatisticamente por meio do Statistic Package Social for Science (SPSS, versão 12.0). A análise comparativa entre o Grupo 1 e o Grupo 2 foi processada a partir do teste $t$ de Student, paramétrico, para amostras independentes. $\mathrm{O}$ valor de significância adotado no presente estudo foi de $p$ menor ou igual a 0,05 (estimadas com $95 \%$ de confiança). Os resultados estão expressos em tabelas, cuja primeira linha de cada mês se refere aos valores obtidos pelo Grupo 1 e a segunda linha ao Grupo 2. É importante observar que o numero de lactentes dos grupos variou a cada mês em função de adesões tardias ao projeto e, também, em caso de faltas nas avaliações observadas, principalmente nos últimos meses de avaliação.

\section{RESULTADOS}

Os resultados de desenvolvimento infantil da população estudada foram analisados a partir do fator de risco prematuridade, comparando-o com o desenvolvimento de crianças sem condições de risco, mês a mês, no primeiro ano de vida. Os desempenhos dos RNs de cada grupo, a partir das avaliações conduzidas com o IPO, foram comparados para cada uma das áreas avaliadas.

Em autocuidado observou-se diferença significante somente para os desempenhos obtidos no mês $4(\mu=6,92 / \mathrm{DP}=2,23$ e $\mu=8,32 /$ $\mathrm{DP}=3,47, \mathrm{p}=0,035)$, quando comparados os
Grupos 1 e 2, respectivamente. Observa-se que, nesta fase, os lactentes têm desempenhos muito parec

Tabela 1: Médias, desvios padrão e resultados do Teste $t$ (2-tailed) na comparação entre os Grupos 1 e 2, na área de linguagem

\begin{tabular}{|c|c|c|c|c|}
\hline & $\mathbf{N}$ & MÉDIA & D P & $p$ \\
\hline \multirow[t]{2}{*}{ mês 1} & 56 & ,6429 & 6723 & \\
\hline & 34 &, 7353 & ,6183 & - \\
\hline \multirow[t]{2}{*}{ mês 2} & 59 &, 8475 & 6902 & \\
\hline & 38 & 1,8158 & 1,3122 &, 000 \\
\hline \multirow[t]{2}{*}{ mês 3} & 57 & 1,5088 & 1,4775 & \\
\hline & 41 & 2,3659 & 1,2401 &, 002 \\
\hline \multirow[t]{2}{*}{ mês 4} & 49 & 2,4490 & 1,5419 & \\
\hline & 38 & 3,4737 & 1,7512 & ,006 \\
\hline \multirow[t]{2}{*}{ mês 5} & 46 & 3,3478 & 1,6629 & \\
\hline & 39 & 4,0513 & 1,8631 & - \\
\hline \multirow[t]{2}{*}{ mês 6} & 43 & 4,2558 & 2,3104 & \\
\hline & 37 & 4,9189 & 2,0052 & - \\
\hline \multirow[t]{2}{*}{ mês 7} & 38 & 5,0263 & 2,3651 & \\
\hline & 31 & 6,5161 & 2,4883 &, 014 \\
\hline \multirow[t]{2}{*}{ mês 8} & 28 & 6,0000 & 2,5240 & \\
\hline & 29 & 7,9310 & 2,9753 & ,011 \\
\hline \multirow[t]{2}{*}{ mês 9} & 26 & 7,4615 & 2,6867 & \\
\hline & 25 & 9,2400 & 2,2226 & ,013 \\
\hline \multirow[t]{2}{*}{ mês 10} & 22 & 9,7273 & 2,3540 & \\
\hline & 23 & 10,0000 & 2,0000 & - \\
\hline \multirow[t]{2}{*}{ mês 11} & 19 & 13,4737 & 10,9360 & \\
\hline & 16 & 11,1250 & 1,6683 & - \\
\hline \multirow[t]{2}{*}{ mês 12} & 15 & 11,7333 & 1,9445 & \\
\hline & 16 & 12,2500 & ,8563 & - \\
\hline
\end{tabular}

Na Tabela 1 observam-se os dados relativos à comparação entre os desempenhos dos RNs no IPO, em linguagem. Nota-se que o Grupo 2, de prematuros, apresentou diferenças significantes para seis dos doze meses avaliados, quando comparado ao Grupo 1 (controle). 
Tabela 2: Médias, desvios padrão e resultados do Teste $t$ (2-tailed) na comparação entre os Grupos 1 e 2, na área de desenvolvimento motor

\begin{tabular}{|c|c|c|c|c|}
\hline \multirow{2}{*}{$\frac{N}{\text { mês } 1}$} & \multicolumn{2}{|c|}{ MÉDIA } & \multirow{2}{*}{$\begin{array}{l}\text { D P } \quad p \\
1,6156\end{array}$} & \\
\hline & 56 & 2,5893 & & \\
\hline & 34 & 3,7647 & 2,0898 &, 007 \\
\hline \multirow[t]{2}{*}{ mês 2} & 59 & 4,5763 & 2,3059 & \\
\hline & 38 & 6,4474 & 2,8541 &, 001 \\
\hline \multirow[t]{2}{*}{ mês 3} & 56 & 7,6429 & 3,9746 & \\
\hline & 40 & 10,1750 & 3,3960 &, 001 \\
\hline \multirow[t]{2}{*}{ mês 4} & 49 & 11,0816 & 4,6852 & \\
\hline & 38 & 17,4474 & 5,5639 &, 000 \\
\hline \multirow[t]{2}{*}{ mês 5} & 46 & 16,5870 & 6,0169 & \\
\hline & 38 & 23,8947 & 6,0126 &, 000 \\
\hline \multirow[t]{2}{*}{ mês 6} & 43 & 23,6512 & 7,5872 & \\
\hline & 37 & 32,1622 & 6,5511 &, 000 \\
\hline \multirow[t]{2}{*}{ mês 7} & 38 & 30,6842 & 8,5932 & \\
\hline & 30 & 39,1000 & 4,8234 &, 000 \\
\hline \multirow[t]{2}{*}{ mês 8} & 28 & 36,2857 & 10,1830 & \\
\hline & 29 & 43,7241 & 7,5918 &, 003 \\
\hline \multirow[t]{2}{*}{ mês 9} & 26 & 41,4231 & 8,2130 & \\
\hline & 25 & 49,9200 & 4,9068 &, 000 \\
\hline \multirow[t]{2}{*}{ mês 10} & 22 & 48,8636 & 6,6210 & \\
\hline & 23 & 53,1304 & 4,4549 &, 016 \\
\hline \multirow[t]{2}{*}{ mês 11} & 19 & 52,8947 & 4,3320 & \\
\hline & 16 & 55,3125 & 2,5747 &, 050 \\
\hline \multirow[t]{2}{*}{ mês 12} & 15 & 55,3333 & 2,4398 & \\
\hline & 16 & 57,1875 & 1,4705 & 018 \\
\hline
\end{tabular}

Em desenvolvimento motor, para o Grupo 2, houve diferenças significantes em todos os meses avaliados, quando comparados ao Grupo 1, conforme mostra a Tabela 2.

Em cognição, para o Grupo 2 houve diferenças significantes em 11 dos 12 meses avaliados, quando comparados ao desempenho do Grupo 1 (Tabela 3).
Tabela 3: Médias, desvios padrão e resultados do Teste $t$ (2-tailed) na comparação entre os Grupos 1 e 2, na área de cognição

\begin{tabular}{|c|c|c|c|c|}
\hline & $\mathbf{N}$ & MÉDIA & D P & $p$ \\
\hline \multirow[t]{2}{*}{ mês 1} & 56 & 5,1964 & 3,4715 & \\
\hline & 34 & 7,9118 & 4,6147 & ,005 \\
\hline \multirow[t]{2}{*}{ mês 2} & 59 & 10,0339 & 4,2587 & \\
\hline & 38 & 12,0263 & 3,8589 & ,019 \\
\hline \multirow[t]{2}{*}{ mês 3} & 57 & 13,0702 & 4,1398 & \\
\hline & 41 & 14,0244 & 3,0289 & - \\
\hline \multirow[t]{2}{*}{ mês 4} & 49 & 15,0612 & 2,7719 & \\
\hline & 38 & 16,3158 & 2,9783 & ,048 \\
\hline \multirow[t]{2}{*}{ mês 5} & 46 & 16,8478 & 1,6729 & \\
\hline & 39 & 18,0769 & 3,3747 & ,043 \\
\hline \multirow[t]{2}{*}{ mês 6} & 43 & 18,4651 & 2,0970 & \\
\hline & 37 & 20,5405 & 2,5451 &, 000 \\
\hline \multirow[t]{2}{*}{ mês 7} & 38 & 20,4737 & 2,3566 & \\
\hline & 31 & 23,0645 & 2,1593 &, 000 \\
\hline \multirow[t]{2}{*}{ mês 8} & 28 & 22,3929 & 2,7533 & \\
\hline & 29 & 24,5172 & 2,6675 &, 005 \\
\hline \multirow[t]{2}{*}{ mês 9} & 26 & 23,7692 & 2,8609 & \\
\hline & 26 & 26,5769 & 2,1573 &, 000 \\
\hline \multirow[t]{2}{*}{ mês 10} & 22 & 25,4545 & 2,2830 & \\
\hline & 23 & 27,8696 & 1,7659 &, 000 \\
\hline \multirow[t]{2}{*}{ mês 11} & 19 & 27,0000 & 1,9149 & \\
\hline & 16 & 28,8750 & 1,3102 &, 002 \\
\hline \multirow[t]{2}{*}{ mês 12} & 15 & 28,1333 & 1,3558 & \\
\hline & 16 & 29,3125 & ,7042 &, 007 \\
\hline
\end{tabular}

Em socialização, para o Grupo 2 o desempenho foi significantemente pior do que o Grupo 1 em 11 dos 12 meses avaliados, conforme mostra a Tabela 4. 
Tabela 4: Médias, desvios padrão e resultados do Teste $t$ (2-tailed) na comparação entre os Grupos 1 e 2, na área de socialização

\begin{tabular}{|c|c|c|c|c|}
\hline & $\mathbf{N}$ & MÉDIA & D P & $p$ \\
\hline & $\mathbf{N}$ & MÉDIA & D P & $p$ \\
\hline \multirow[t]{2}{*}{ mês 1} & 56 & 3,2321 & 2,2882 & \\
\hline & 34 & 4,6471 & 2,6272 &, 012 \\
\hline \multirow[t]{2}{*}{ mês 2} & 59 & 6,6949 & 3,4153 & \\
\hline & 38 & 8,3684 & 3,5138 & ,023 \\
\hline \multirow[t]{2}{*}{ mês 3} & 57 & 10,3158 & 4,0583 & \\
\hline & 41 & 12,4878 & 3,5009 & ,006 \\
\hline \multirow[t]{2}{*}{ mês 4} & 49 & 14,0816 & 3,8936 & \\
\hline & 38 & 17,4737 & 4,3974 &, 000 \\
\hline \multirow[t]{2}{*}{ mês 5} & 46 & 15,8913 & 5,0913 & \\
\hline & 39 & 20,6667 & 3,9225 &, 000 \\
\hline \multirow[t]{2}{*}{ mês 6} & 43 & 21,2326 & 4,0815 & \\
\hline & 37 & 24,5946 & 2,8133 &, 000 \\
\hline \multirow[t]{2}{*}{ mês 7} & 38 & 24,2368 & 3,3404 & \\
\hline & 31 & 26,7419 & 2,5162 &, 001 \\
\hline \multirow[t]{2}{*}{ mês 8} & 28 & 25,2500 & 3,2956 & \\
\hline & 29 & 28,1724 & 2,4064 &, 000 \\
\hline \multirow[t]{2}{*}{ mês 9} & 26 & 26,7692 & 2,4217 & \\
\hline & 26 & 30,0769 & 1,9783 &, 000 \\
\hline \multirow[t]{2}{*}{ mês 10} & 22 & 29,2273 & 2,4868 & \\
\hline & 23 & 31,0000 & 1,7838 & ,009 \\
\hline \multirow[t]{2}{*}{ mês 11} & 19 & 30,7368 & 1,8209 & \\
\hline & 16 & 31,8750 & 1,4083 &, 045 \\
\hline \multirow[t]{2}{*}{ mês 12} & 15 & 31,6000 & 1,8439 & \\
\hline & 16 & 32,3125 & 1,2500 & \\
\hline
\end{tabular}

Os dados obtidos a partir das comparações entre os Grupos 1 e 2, em todas as áreas avaliadas, mostra que, em $68 \%$ das possibilidades de avaliação, o desempenho do Grupo 2 (prematuros) foi significantemente diferente do Grupo 1, para a grande maioria das comparações.

\section{DISCUSSÃO}

Os resultados obtidos apontaram que os RNs prematuros (Grupo 2) apresentaram desempenho significantemente diferente do Grupo 1 em $68 \%$ das análises conduzidas, com destaque para as áreas de desenvolvimento motor, cognição e socialização. Tais resultados são condizentes com os obtidos na literatura que apontam a prematuridade como uma das condições proximais mais prejudiciais ao desenvolvimento de lactentes ${ }^{32,20,7}$ com consequências para $\mathrm{o}$ desenvolvimento de outras habilidades, principalmente na idade escolar ${ }^{21,24,25,26}$.

Castro et al. ${ }^{32}$ encontraram prejuízos no desenvolvimento de RNs prematuros relacionados à idade gestacional. Barbosa, Formiga e Linhares $^{33}$ avaliaram 21 recém-nascidos prétermo (com 32 semanas) com o NAPI (Neurobehavioral Assessment of the Preterm Infant). Os resultados indicaram desempenho abaixo do esperado para quatro das sete categorias avaliadas. Resegue, Piccini e Silva ${ }^{7}$ também identificaram, em seus estudos, prejuízos para as crianças de baixo peso ao nascimento, mas não separaram os RNs prematuros, que também nascem com baixo peso, o que corrobora os achados deste estudo, no que diz respeito ao baixo desempenho dos prematuros.

Os dados obtidos em linguagem são corroborados pelos achados de Lamônica e Picolini ${ }^{30}$ que, a partir de dados obtidos com o IPO, encontraram diferenças significativas aos seis e 12 meses de idade, mas não confirmaram os resultados de autocuidado que, no mesmo estudo, encontraram diferenças significativas aos seis e aos 12 meses e, neste estudo, a única diferença observada foi aos quatro meses de idade. Fraga, Linhares, Carvalho e Martinez ${ }^{34}$ também encontraram, numa população de prematuros avaliados aos 12 meses, desempenho em linguagem abaixo do esperado. Pereira e Funayama ${ }^{35}$ observaram atrasos na linguagem receptiva de RNs prematuros, na faixa de três a 12 meses, o que confirma os 
achados deste estudo em que a linguagem receptiva é avaliada em cognição, área na qual o Grupo 2 apresentou 11 diferenças significativas, quando comparado ao Grupo 1.

A prevenção secundária que objetiva impedir a evolução de possíveis sequelas em indivíduos já expostos à condição de risco para o desenvolvimento, pode ser implementada, entre outras possibilidades, pela avaliação precoce dos efeitos dos fatores de risco e a pela condução de programas de estimulação precoce. A partir de avaliações do desenvolvimento infantil é possível conduzir intervenções que promovam o desenvolvimento, minimizando fatores de risco.

$\mathrm{Na}$ avaliação do desenvolvimento, um dos aspectos a ser considerado é a utilização ou não da idade corrigida. Neste estudo optouse pela utilização, nas análises, das idades cronológicas das crianças. É preciso atentar para o papel das influências ambientais para responder se o desenvolvimento do lactente fora do útero é o mesmo do que seria antes do nascimento. Dependendo da intensidade da prematuridade, oportunidades deixariam de ser oferecidas aos RNs em programas de estimulação porque estariam sendo considerados com dois ou até três meses menos. É possível que o lactente apresente comportamentos típicos da sua idade cronológica mais rapidamente. Entretanto, estudos comparativos devem ser conduzidos para analisar a pertinência das análises e os desdobramentos nos projetos de estimulação precoce.

Bailey $\mathrm{Jr}^{36}$ enfatiza que tais programas devem se preocupar com o que a criança tem de aprender e como essa aprendizagem pode ser facilitada. Para ele a ênfase deve estar na funcionalidade das habilidades estimuladas, para que possa se adaptar às demandas do seu ambiente. Sugere ainda, que a estimulação precoce para RNs prematuros, deve providenciar, desde estratégias para estimulação durante a estadia do recém-nascido no hospital, mesmo que na UTI, até estratégias para acompanha- mento do desenvolvimento da criança pelo menos até a idade escolar ${ }^{36}$. Para Linhares ${ }^{37}$ a estimulação precoce tem o papel de interromper ou, pelo menos, minimizar os efeitos dos fatores de risco presentes no desenvolvimento da criança. Considerando o RN prematuro, é provável que quanto mais precoce e regulada às suas necessidades for a intervenção, mais efetiva será para o seu desenvolvimento.

Os participantes deste estudo são oriundos de um projeto de extensão que avalia, mensalmente, os lactentes e orienta os pais. A análise conduzida mostrou que RNs prematuros apresentaram defasagens importantes na maioria das áreas avaliadas, durante todo o primeiro ano de vida.

O presente estudo trouxe respostas e indagações, apontando para a necessidade de se considerar as variáveis distais, meio-proximais e proximais como sendo indicadores de quais crianças deveriam, obrigatoriamente, participar de programas de estimulação precoce que monitorariam seu desenvolvimento, com maior atenção para os prematuros. Todavia, é preciso investimentos em instrumentos de avaliação adequados e, se possível, padronizados que permitam monitorar o desenvolvimento de crianças de risco. É importante, também, investir em programas de intervenção precoce que, se não eliminam, podem minimizar os efeitos de variáveis prejudiciais ao desenvolvimento, proporcionando indivíduos sadios e adaptados.

Os resultados obtidos com esta pesquisa permitem analisar o efeito da prematuridade no desenvolvimento infantil e a implementação de projetos, focalizando aquelas condições mais prejudiciais ao desenvolvimento infantil, por um período que excederia ao primeiro ano de vida. Para gerar resultados confiáveis, o instrumento de avaliação do desenvolvimento de lactentes deveria possibilitar a identificação da ocorrência do comportamento, sob determinadas condições, com critérios bem estabelecidos, que permitissem a análise funcional dos mesmos. 
Em caso de ausência, tais informações, por observação ou relato de cuidadores, daqueles comportamentos com pouca chance de aparecer em situações de avaliação, permitiriam a organização de contingências que aumentariam a possibilidade de ocorrência no ambiente natural da criança, possibilitando um desenvolvimento adequado ao esperado para sua idade cronológica. Os resultados obtidos com este estudo permitiram constatar, para a população estudada, o efeito da prematuridade no desen-

\section{REFERÊNCIAS}

1. Bijou SW, Baer DM. Psicologia del desarrollo infantil: teoria empírica y sistemática de la conducta. México: Editorial Trillas, 1976.

2. Weber LND. Interações entre família e desenvolvimento. In: WEBER, LND, org. Família e desenvolvimento: visões multidisciplinares. Curitiba: Editora Juruá; 2008. p. 9-20.

3. Rosales-Ruiz J, Baer DM. Behavioral cusps: a developmental and pragmatic concept for behavior analysis. JABA, 1997;30 (3);533-544.

4. Matos MA. A medida do ambiente de desenvolvimento infantil. Psicologia. 1983;9(1):5-18.

5. Oliveira EA. Modelos de risco na Psicologia do desenvolvimento. Psicologia: Teoria e pesquisa. 1998;14 (1):19-26.

6. Halpern R, Figueiras ACM. (2004). Influências ambientais na saúde mental da criança. J.Pediatr, 2004;80 (2):104-110.

7. Resegue R, Puccini RF, Silva EMK. Risk factors associated with developmental abnormalities among high-risk children attended at a multidisciplinary clinic. $\mathrm{Br}$ Med J, 2008;126(1):4-10.

8. Resegue R, Puccini RF, Silva EMK. Fatores de risco associados a alterações no desenvolvimento da criança. J.Pediatr, 2007;29(2):117-128. volvimento de lactentes no primeiro ano de vida. Os mesmos indicam a necessidade de implementação de projetos de intervenção específicos que devem ser oferecidos precocemente e além do primeiro ano de vida, com o objetivo de minimizar os efeitos posteriores da prematuridade.

No entanto, dado o pequeno número de participantes, principalmente nos meses finais de avaliação, os resultados devem ser vistos com cautela.

9. Haynes SN, O'Brien WO. Functional analysis in behavior therapy. Clinical Psychology Review, 1990;10:649-668.

10. Calais SL, Bolsoni-Silva AT. Alcance e limites das técnicas comportamentais: algumas considerações. In: Cavalcante MR, org. Análise do Comportamento: Avaliação e intervenção. São Paulo: Editora Roca; 2008. p. 15-31.

11. Meyer SB. O conceito de análise funcional. In: Delitti M, org. Sobre comportamento e cognição: a prática da análise do comportamento e da terapia cognitiva comportamental. Santo André: ARBytes; 2001. p. 29-34.

12. CID-10. Classificação Estatística Internacional de Doenças e Problemas Relacionados à Saúde. 10a rev. (v. 1). São Paulo: Universidade de São Paulo, 1997.

13. Rugolo LMSS. Crescimento e desenvolvimento a longo prazo do prematuro extremo. J. Pediatr, 2005;81(1, Supl.):101 110.

14. Gianini NM, Vieira AA, Moreira EL. Avaliação dos fatores associados ao estado nutricional na idade corrigida de termo em recém-nascidos de muito baixo peso. J.Pediatr, 2005; 81(1):34-40.

15. Manacero S, Nunes ML. Avaliação do desempenho motor de prematuros nos primeiros meses de vida na Escala Motora Infantil de Alberta (AIMS). J.Pediatr, 2008;84 (1):53-59. 
16. Batista E. O bebê prematuro e o desenvolvimento do comportamento no primeiro ano de vida, Psicologia: Reflexão e Crítica/ Psychology, 2009 [acesso em 26 de maio de 2009]; 22(1). Resumo disponível em www.scielo.br/scielo.php-_script_sci _serial_pid_0102-7972.

17. Goyen TA, Lui K. Longitudinal motor development of apparently normal highrisk infants at 18 months, 3 and 5 years. Early Human Development, 2002;70(12):103-115.

18. Hadders-Algra M. General movements: A window for early identification of children at high risk for developmental disorders. J. Pediat, 2004;145(2, Supl):12-18.

19. Magalhães LC, Barbosa VM, Paixão ML, Figueiredo EM, Gontijo APB. Acompanhamento ambulatorial do desenvolvimento de recém-nascidos de alto risco: características da população e incidência de seqüelas funcionais. Revista Paulista de Pediatria, 1998;16:191-196.

20. Magalhães LC, Wendling PC, Paixão ML, Mancini MC, Barbosa VM. Estudo comparativo sobre o desempenho perceptual e motor na idade escolar em crianças nascidas pré-termo e a termo. Arq. Neuro Psiquiatr, 2003;61:250-255.

21. Carvalho AEV, Linhares MBM, Martinez FE. História de desenvolvimento e comportamento de crianças nascidas pré-termo e baixo peso $(<1500$ grs $)$. Psicologia: Reflexão e Crítica, 2001;14(1):1-33.

22. Chermont AG, Cunha MS, Sales LMM, Moraes AN, Malveira SS. Avaliação do desenvolvimento pela escala de Denver II, de recém-nascidos prematuros de baixo peso. Revista CEFAC, 2005;19(2):59-66.

23. Mancini MC, Megale L, Brandão MB, Melo APP, Sampaio RF. Efeito moderador de risco social na relação entre risco biológico e desempenho funcional infantil. Rev. Bras. Saude Mater. Infant., 2004;4 (1): 25-34.
24. Linhares MBM, Carvalho AEV, Bordin MBM, Chimello JT, Martinez FE, Jorge SM. Prematuridade e muito baixo peso como fatores de risco ao desenvolvimento da criança. Cadernos de Psicologia e Educação - Paidéia, 2000;10(12):60-69.

25. Martins IMB, Linhares MB, Martinez FE. Indicadores de desenvolvimento na fase pré-escolar de crianças nascidas pré-termo, Psicologia em Estudo, 2005;10(2):235-243.

26. Méio MDBB, Lopes CS, Morsch DS. Fatores prognósticos para o desenvolvimento cognitivo de prematuros de muito baixo peso. Revista de Saúde Pública, 2003;37 (3):311-318.

27. Souza R, Andrade CRF. Perfil da fluência de crianças prematuras. J.Pediatr, 2004;26 (2):90-96.

28. Nunes LRDP, Sisdelli RO, Fernandes RLC. O valor dos testes de bebês e suas implicações para a Psicologia do Desenvolvimento. Revista Brasileira de Educação Especial, 1995;5:107-125.

29. Williams LCA, Aiello ALR. O Inventário Portage Operacionalizado: intervenção com famílias. São Paulo: Memnon/ FAPESP; 2001.

30. Lamônica DAC, Picolini MM. Habilidades do desenvolvimento de prematuros. Revista CEFAC, 2008 [acesso em 06 de junho de 2009]; Disponível em: http:// www. scielo.br/scielo.php?script=sciarttex\&pid=S1516.

31. Rodrigues OMPR. O Inventário Portage Operacionalizado e o desenvolvimento de bebês. Tese de Livre Docência. Universidade Estadual Paulista Julio de Mesquita Filho (UNESP), Bauru/SP, 2009.

32. Castro A G, Lima MC, Aquino RR, Eickmann SH. Desenvolvimento do sistema sensório motor oral e motor global em lactentes pré-termo. Pró-Fono Revista de Atualização Científica, 2007;19 (1):29-38. 
33. Barbosa VC, Formiga CKMR, Linhares MBM. Avaliação das variáveis clínicas e neurocomportamentais de recém-nascidos pré-termo. Rev. Bras. Fisioter, 2007;11 (4):275-281.

34. Fraga DA, Linhares MBM, Carvalho AEV, Martinez FE. Desenvolvimento de bebês prematuros relacionado a variáveis neonatais e maternas. Psicologia em Estudo, 2008; 13 (2):335-344.

35. Pereira MR, Funayama CAR. Avaliação de alguns aspectos da aquisição e desenvolvimento da linguagem de crianças nascidas pré-termo. Arq. Neuro - Psiquiatr, 2004; 62 (3):641-648.
36. Bailey Jr DB. Evaluating the effectiveness of curriculum alternatives for infants an preschoolers at high risk. In: Guralnick MJ, org. The effetiviness of early intervention Baltimore: Paul Brookes Publishing Co, 1997. p. 227-248.

37. Linhares MBM. Estresse, resiliência e cuidado no desenvolvimento de neonatos de alto risco In: Mendes EG, Almeida MA, Williams LCA, orgs. Temas em Educação Especial: Avanços recentes. São Carlos: EDUFSCar, 2004. p. 315-324.

Recebido em: 10/jul./10 Modificado em: 16/ago./10 Aceito em: 22/dez./10 